\title{
Clinical Characteristics, Management, and Outcomes of the Patients With ST-Segment Elevation Myocardial Infarction Before and During the COVID-19 Pandemic
}

\author{
Nadya Almatrooshia, c, Zafar Khan ${ }^{\mathrm{b}}$, Manju Chakkittayila, Samir AlJaabari ${ }^{\mathrm{b}}$, \\ Yara Elyazori $^{\mathrm{a}}$, Seema Nour ${ }^{\mathrm{b}}$, Abdul Majeed Alzubaidi ${ }^{\mathrm{a}}$
}

\begin{abstract}
Background: The first case of coronavirus disease 2019 (COVID-19) in the United Arab Emirates (UAE) was reported on January 29, 2020. Various anecdotal reports suggest that fewer patients with ST-segment elevation myocardial infarction (STEMI) have been presented to the hospital during the COVID-19 pandemic. In this study, we examined the patient characteristics, in-hospital management, and outcomes of the patients with STEMI before and during the COVID-19 pandemic in Abu Dhabi, UAE.

Methods: We analyzed the data retrospectively from January 1, 2019 to December 31, 2020 and stratified the same into two periods: before the pandemic (January 1, 2019 to January 28, 2020) and during the pandemic (January 29, 2020 to December 31, 2020). The patient-level data for the two periods were compared to identify the differences in the patient characteristics, in-hospital management, and 30-day mortality outcomes of the patients with STEMI.

Results: During the COVID-19 pandemic, the number of STEMI cases was lower $(n=234)$ than before the pandemic $(n=266)$, and 11 patients (4.7\%) tested positive for COVID-19 during their hospital encounter. In comparison to before the pandemic, patients with STEMI were significantly younger (mean age: 50.39 years, standard deviation (SD) \pm 10.32 years), less likely to present to the emergency department (ED), and more likely to have histories of hypercholesterolemia $(\mathrm{P}<0.001)$ and coronary artery disease $(\mathrm{P}<0.001)$. Significantly shorter door-to-balloon time $(56.6 \pm 27.26 \mathrm{~min}, \mathrm{P}<0.001)$ and decreased length of stay $(4.7 \pm 6.8$ days, $\mathrm{P}<0.001)$ were observed during the pandemic. However, no considerable difference in the 30 day mortality was observed in 2020 as compared to before the pandemic $(2.1 \%$ versus $3.0 \%$, respectively).
\end{abstract}

Manuscript submitted September 27, 2021, accepted October 19, 2021

Published online December 28, 2021

aDepartment of Cardiology, Sheikh Shakboot, Abu Dhabi, United Arab Emirates

bDepartment of Cardiology, Sheikh Khalifa Medical City, Abu Dhbai, United Arab Emirates

'Corresponding Author: Nadya Almatrooshi, Department of Cardiology, Sheikh Shakboot, Abu Dhabi, United Arab Emirates.

Email: nmatroshi@seha.ae

doi: https://doi.org/10.14740/cr1275
Conclusions: Hospital presentation for the patients with STEMI decreased during the COVID-19 pandemic, with significant differences in the door-to-balloon time and length of hospital stay. This indicates that the hospital systems of care continued to operate with higher efficiency during the COVID-19 pandemic than before the pandemic in the UAE.

Keywords: Coronavirus disease; Pandemic; STEMI; Mortality

\section{Introduction}

ST-segment elevation myocardial infarction (STEMI) is a medical emergency that requires immediate medical evaluation for the patients experiencing symptoms and guideline-recommended timely revascularization to improve the outcomes $[1,2]$. However, anecdotal evidence suggests a decrease in the STEMI cases and delay in the emergency evaluation during the coronavirus disease 2019 (COVID-19) pandemic period [3-5].

Since the first case of COVID-19 infection reported in the United Arab Emirates (UAE) on January 29, 2020, there has been a steep upsurge in the number of confirmed cases and attributable deaths [6]. There are several caveats unique to the management of STEMI during the COVID-19 pandemic, particularly the patient's reluctance to engage with medical services due to the apprehension of getting infected while in the hospital [7]. Additionally, there has been noted a delay in the patient presentation, referral, and emergency medical services (EMSs) to transport the STEMI patients to the treatment facility [7-9]. A number of reports worldwide delineate the presentation, management, and outcomes in the patients with STEMI admitted during the pandemic [7-12]. However, to the best of our knowledge, the evidence regarding the detrimental effects of the pandemic and restrictions of public life on the presentation, management, and the early outcomes of the patients with STEMI from the UAE is insufficient. Therefore, the primary aim of this study was to assess and compare the clinical characteristics, in-hospital management, and outcomes of the patients with STEMI before and during the COVID-19 pandemic era.

We hypothesized that the number of patients with STEMI and their pattern of seeking care, the severity of presenting symptoms, and acute care intervention metrics would differ 
before and during the COVID-19 pandemic. The cut-off date, January 29,2020, was selected as that is when the first case of COVID-19 in the UAE was reported.

\section{Materials and Methods}

This is a retrospective, multicenter observational study on the patients with STEMI consecutively admitted in the two tertiary care government hospitals equipped with 24 -h percutaneous coronary intervention (PCI) and STEMI reperfusion strategies in Abu Dhabi, UAE. Electronic medical records were screened using the Cerner ${ }^{\circledR}$ electronic database to identify the patients with STEMI treated under Abu Dhabi health services (SEHA) in the UAE. Eligible patients were above 18 years of age, hospitalized, or presented to the emergency department (ED) with STEMI from January 1, 2019 to December 31, 2020. Patients admitted during the COVID-19 pandemic from January 29, 2020 to December 31, 2020 were compared with the before pandemic period from January 1, 2019 to January 28, 2020.

\section{Data collection}

The following variables were extracted from the electronic medical records for the patient-level analysis: age, sex, nationality, history of smoking, previous history of coronary artery disease, PCI, coronary artery bypass graft, comorbidities, past medication history, arrival method (personal transport, EMS usage, or in-hospital STEMI code or transferred from another facility). Furthermore, the presenting symptoms (categorized into chest pain or shortness of breath), vitals and clinical features such as arrhythmias, left ventricular ejection fraction (in terms of percentage), electrocardiographic findings, and other parameters were collected. The laboratory results including serum creatinine, hemoglobin, cardiac enzymes, etc., were collected. Angiographic and procedural details included identifying culprit as well as non-culprit vessels, segments, branches, requirement of staged PCI, and the number of stents. Timing variables, for instance, the emergency door-to-balloon (D2B) time were ascertained. Besides, we measured the duration of the in-hospital stay, discharge outcomes, and 30-day mortality outcomes from the day of discharge of patients with STEMI were collected.

The Department of Health (DOH), Ministry of Health approved the study protocol (DOH/CVDC/2020/1877); and the anonymity of the patients was maintained to protect their respective identities. The study was conducted in compliance with the ethical standards of the responsible institution on human subjects as well as with the Helsinki Declaration.

\section{Statistical analyses}

Statistical analyses were performed using the SPSS software ver. 22.0 (IBM, Armonk, NY, USA). The categorical variables were presented as absolute numbers and percentages.
The numerical data were presented as mean \pm standard deviation (SD). The categorical variables were compared using the Chi-square test, and the Student's $t$-test or analysis of variance (ANOVA) was used for continuous variables. The 30day survival curves were constructed using the Kaplan-Meier methodology and compared using the log-rank test. Statistical analysis was two-tailed, and a $\mathrm{P}$ value $<0.05$ was considered statistically significant.

\section{Results}

A total of 500 STEMI cases were registered in two tertiary care facilities in Abu Dhabi from January 2019 to December 2020. The baseline characteristics of 266 patients $(53.2 \%)$ in 2019 (before pandemic) and 234 subjects (46.8\%) during the COVID-19 pandemic are enumerated in Table 1. A total of 11 patients $(4.7 \%)$ of the pandemic cohort were tested positive for COVID-19 during their hospital encounter. The STEMI patients during the pandemic period were more likely to have a prior diagnosis of hypercholesterolemia $(\mathrm{P}<0.001)$ and coronary artery disease $(\mathrm{P}<0.001)$. These patients were less likely to present to $\mathrm{ED}(\mathrm{P}<0.001)$, had a higher heart rate at admission $(\mathrm{P}=0.028)$, arrhythmias $(\mathrm{P}=0.015)$, and a higher proportion of patients had inferior infract STEMI (54.3\%). There were no significant differences in the age, sex, pre-STEMI antiplatelet or anticoagulant, statin use, and history of diabetes mellitus, smoking, chest pain symptoms, and ejection fraction values.

Regarding the angiographic characteristics and outcomes, we observed a significantly shorter D2B of $56.6 \pm 27.26 \mathrm{~min}$ during the pandemic period, lower staged-PCI procedures, and shorter length of hospital stay. During the COVID-19 pandemic, $90 \%$ of the patients were discharged to home, $7.3 \%$ of the STEMI patients were referred to long-term care, and two patients $(0.9 \%)$ had ST-segment re-evaluation (re-infarction). The mortality rate was $2.6 \%(\mathrm{n}=13)$, and low mortality $(2.1 \%)$ was observed during the pandemic period (Table 2).

The Kaplan-Meier survival curve denoted increased 30day mortality in 2019 than during the COVID-19 pandemic (log-rank $\mathrm{P}=0.054$ ) (Fig. 1). Cox proportional hazard analysis calculated a hazard ratio of 0.35 ( $95 \%$ confidence interval $(\mathrm{CI}): 1.02-1.21, \mathrm{P}=0.098)$ during the pandemic period as compared to that before it.

\section{Discussion}

This paper documents the significant changes in the hospital presentation, atherogenic characteristics, and in-hospital management of patients with STEMI before and during the COVID-19 pandemic in Abu Dhabi, UAE. Together with these changes, the 30-day mortality was observed to decline in patients with STEMI during the COVID-19 pandemic. The overall 12\% decrease in the STEMI cases during the pandemic period relative to 2019 in our study is comparable to that described in recent reports from the USA [3], Italy [5], Spain [13], and Austria [14]. With regard to the patient characteristics, it 
Table 1. Baseline Characteristics and In-Hospital Management of Patients With STEMI Before and During COVID-19 Pandemic

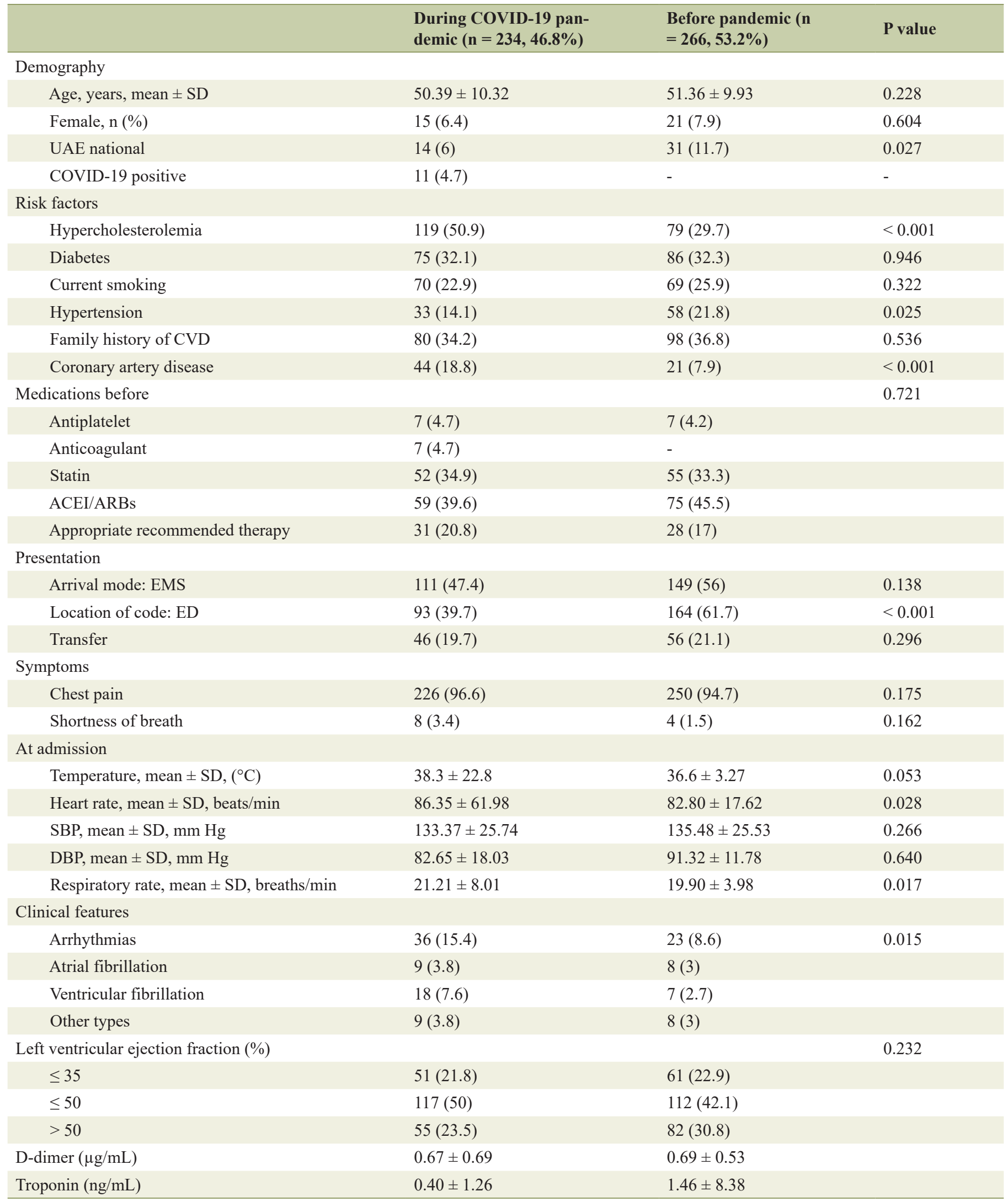


Table 1. Baseline Characteristics and In-Hospital Management of Patients With STEMI Before and During COVID-19 Pandemic (continued)

\begin{tabular}{|c|c|c|c|}
\hline & $\begin{array}{l}\text { During COVID-19 pan- } \\
\text { demic }(n=234,46.8 \%)\end{array}$ & $\begin{array}{l}\text { Before pandemic (n } \\
=266,53.2 \%)\end{array}$ & P value \\
\hline Creatinine (mg/dL) & $1.03 \pm 0.72$ & $1.03 \pm 0.64$ & 0.064 \\
\hline Location of infraction & & & 0.014 \\
\hline Anterior wall & $98(41.9)$ & $133(50.2)$ & \\
\hline Lateral & $2(0.9)$ & $12(4.5)$ & \\
\hline
\end{tabular}

COVID-19: coronavirus disease 2019; STEMI: ST-segment elevation myocardial infarction; UAE: United Arab Emirates; SD: standard deviation; CVD: cardiovascular disease; EMS: emergency medical service; ED: emergency department; ACEI: angiotensin-converting enzyme inhibitor; ARBs: angiotensin-receptor blockers; ECG: electrocardiogram.

is worth noting that the age of patients with STEMI declined, and there was a significant decrease in patients presented to ED during the pandemic, consistent with the anecdotes and the previously reported data suggesting a declining trend in the presentation of non-COVID-19-related medical conditions [711], including STEMI. During the COVID-19 pandemic, the government implemented orders to stay at home, and patients may have been less inclined to present to hospitals because of the fear of contracting COVID-19. Furthermore, a decrease in commutes intuitively correlates with lower anxiety and fewer activities to promote atherosclerotic plaque rupture [15]. Zitelny et al [15] hypothesized that a number of patients with

Table 2. Angiographic Characteristics and Outcomes

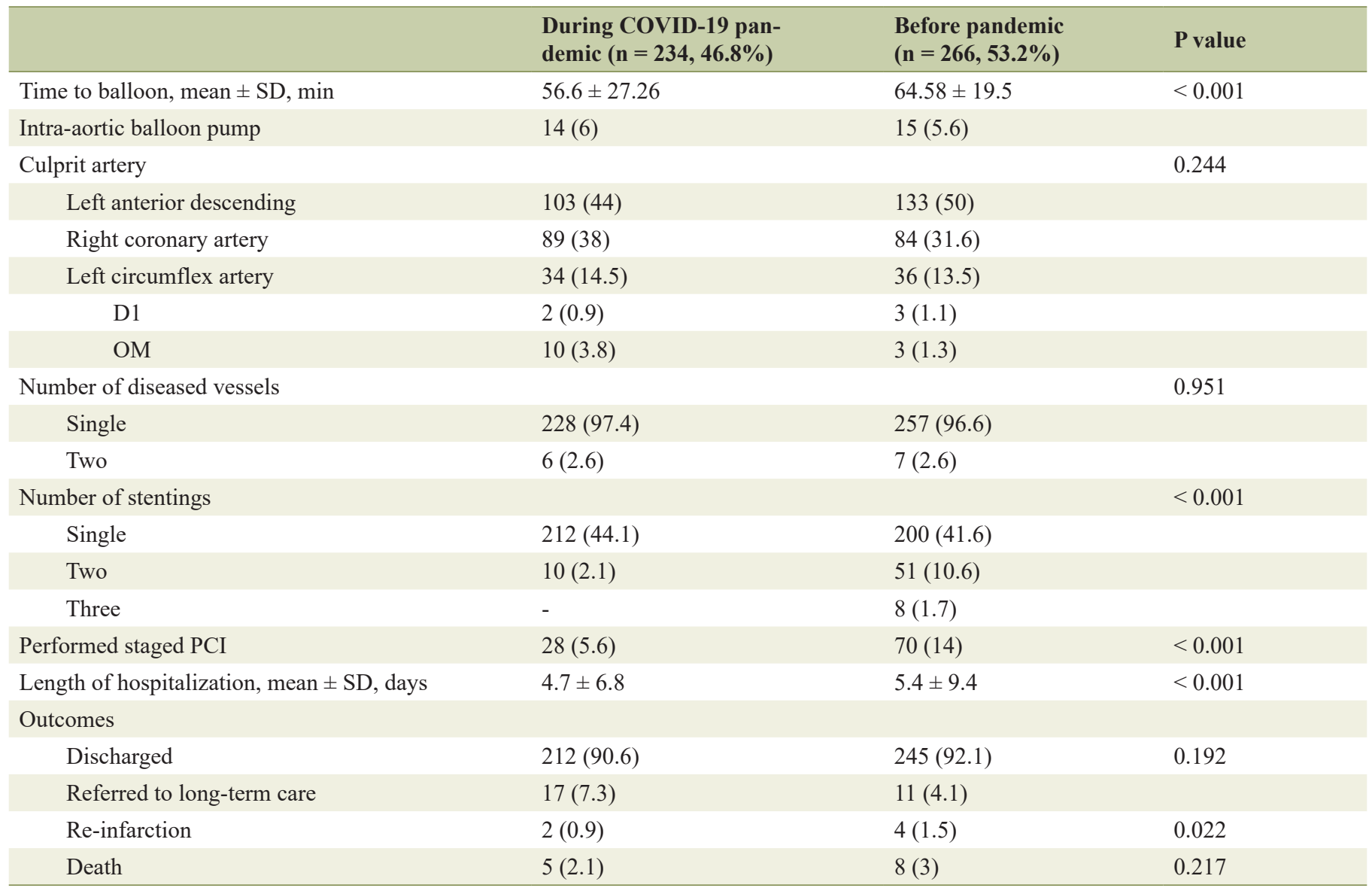

COVID-19: coronavirus disease 2019; SD: standard deviation; PCl: percutaneous coronary intervention; D1: first diagonal; OM: obtuse marginal. 


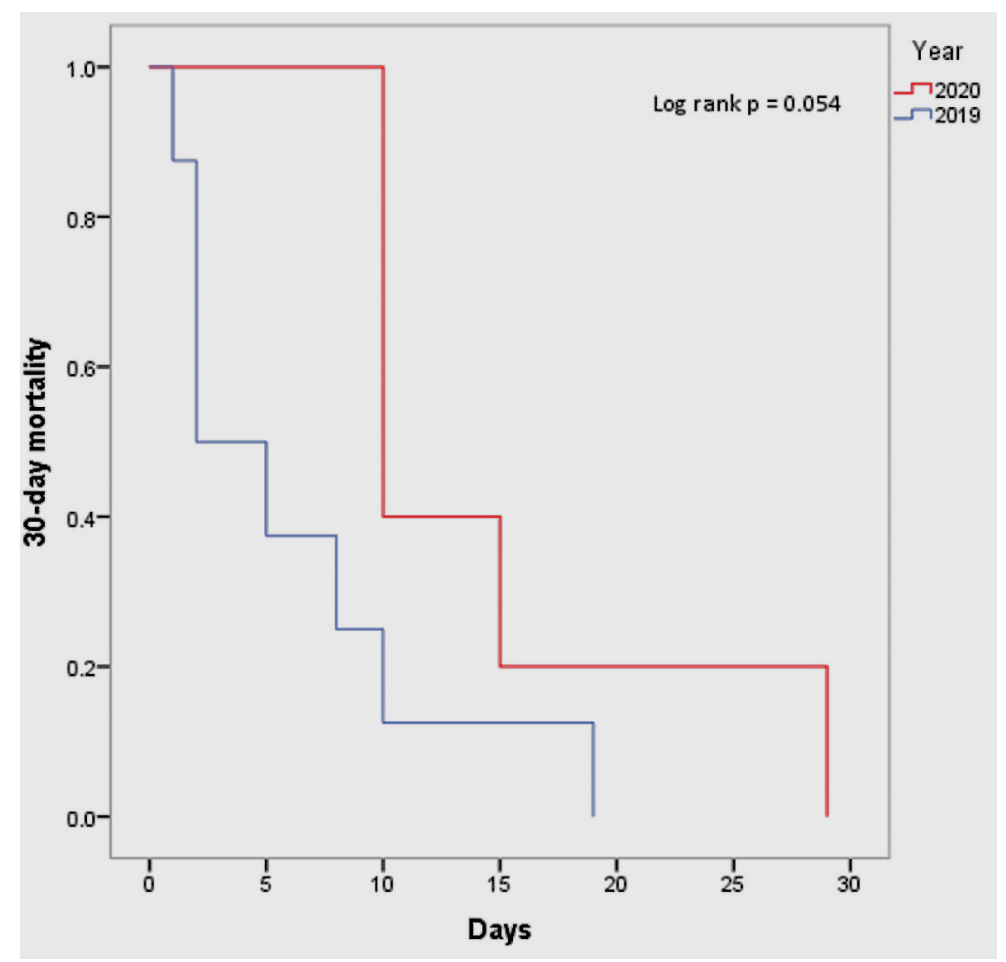

Figure 1. Kaplan-Meier time to event model for 30-day mortality in patients with STEMI in 2019 - 2020. STEMI: ST-segment elevation myocardial infarction.

risk factors for STEMI might dismiss the chest pain as benign relative to the risk of contracting COVID-19 at a hospital [15].

With our observations, we found that the pandemic-epoch patients were more likely to have hypercholesterolemia and coronary artery disease histories and had higher heart rates during hospitalization. There was no difference in the use of EMS services between the before- and during-pandemic periods. Interestingly, there was a statistically significant difference in the D2B time, indicating that hospital systems of care continued to operate more efficiently during the COVID-19 pandemic.

Concrete reports detailing the angiographic findings and outcomes of the patients with STEMI during the COVID-19 pandemic are limited. Daoulah et al [16] evaluated the patients with STEMI from 2018 to 2020 in Saudi Arabia. Their findings demonstrated that the majority of these patients had an infarction in the anterior wall, at least one culprit vessel, and $24.4 \%$ had two or more stents. Chew et al [17] retrospectively evaluated 303 STEMI patients admitted in Singapore between October 2019 and March 2020. During the pandemic, the median D2B time was 55 min (range: 39 - $74 \mathrm{~min}$ ) during the COVID-19 outbreak response.

One of the most remarkable findings of this study is that although there was a significant change in the clinical and atherogenic characteristics in the patients with STEMI, the 30day mortality continued to decrease in the patients with STEMI during the COVID-19 pandemic. However, we did not find a difference in the 30-day mortality between the two periods. We believe that this decline may be driven chiefly by the active utilization of EMS, short reperfusion time, and reduction in the hospital length of stay that may increase post-discharge surveillance. Our results are analogous to and relatively lower than a recent study in the USA that indicated a $7.9 \%$ absolute decrease in the 30-day mortality in the patients with STEMI [18], whereas in England, a group of researchers observed a median reduction in the length of stay of STEMI patients from 3 days to 2 days [19].

\section{Limitations}

Herein, we acknowledge several limitations of this study. First, this was a retrospective, pre- and during COVID-19 analysis, and was delimited by the design constructs of this study. Second, the population was predominantly heterogeneous, and therefore, our results cannot be straightforwardly generalized. Third, we included only two tertiary hospitals in Abu Dhabi, restraining the plausible generalizability to other health facilities in the UAE. Furthermore, some of the associations between the patient characteristics and management may have an implied causal relationship due to confounding. Our study was not powered to detect this subtle difference. Lastly, the possibility of missing critical unmeasured variables could have been introduced owing to the retrospective nature of this study.

\section{Conclusions}

We observed a decrease in the hospital presentation for the patients with STEMI during the COVID-19 pandemic, with significant differences in the D2B time and length of hospital 
stay. This indicates that the hospital systems of care continued to operate with high efficacy during the COVID-19 pandemic in the UAE. However, no significant difference in the 30-day mortality was observed before and during the pandemic, which implies that the care strategies after discharge are crucial to improve the STEMI outcomes.

\section{Acknowledgments}

The authors want to acknowledge the Cardiology Department together with the Cath Lab team at Sheikh Khalifa Medical City and Sheikh Shakbout Medical City.

\section{Financial Disclosure}

No funding is granted for this project.

\section{Conflict of Interest}

None to declare.

\section{Informed Consent}

No informed consent was needed as this was a retrospective chart review (IRB Ref : DOH/CVDC/2020/1877).

\section{Author Contributions}

Dr. Abdul Majeed Alzubaidi and Dr. Nadya Almatrooshi had the idea for and designed the study and had full access to all the data. Manju Chakkittayil extracted the data from the electronic medical records. Dr. Yara Elyazori performed the analytical analysis. Dr. Abdul Majeed Alzubaidi and Dr. Nadya Almatrooshi mainly wrote the article with support from Dr. Zafar Khan, Dr Samir AlJaabari, Dr. Yara Elyazori, and Dr. Seema Nour. All authors provided critical feedback and contributed to the final article.

\section{Data Availability}

The authors declare that data supporting the findings of this study are available within the article.

\section{References}

1. O'Gara PT, Kushner FG, Ascheim DD, Casey DE, Jr., Chung MK, de Lemos JA, Ettinger SM, et al. 2013 ACCF/ AHA guideline for the management of ST-elevation myocardial infarction: a report of the American College of Cardiology Foundation/American Heart Association Task Force on Practice Guidelines. J Am Coll Cardiol. 2013;61(4):e78-e140.

2. Ibanez B, James S, Agewall S, Antunes MJ, BucciarelliDucci C, Bueno H, Caforio ALP, et al. 2017 ESC Guidelines for the management of acute myocardial infarction in patients presenting with ST-segment elevation: The Task Force for the management of acute myocardial infarction in patients presenting with ST-segment elevation of the European Society of Cardiology (ESC). Eur Heart J. 2018;39(2):119-177.

3. Garcia S, Albaghdadi MS, Meraj PM, Schmidt C, Garberich R, Jaffer FA, Dixon S, et al. Reduction in ST-segment elevation cardiac catheterization laboratory activations in the United States during COVID-19 pandemic. J Am Coll Cardiol. 2020;75(22):2871-2872.

4. Solomon MD, McNulty EJ, Rana JS, Leong TK, Lee C, Sung SH, Ambrosy AP, et al. The COVID-19 pandemic and the incidence of acute myocardial infarction. N Engl J Med. 2020;383(7):691-693.

5. De Filippo O, D'Ascenzo F, Angelini F, Bocchino PP, Conrotto F, Saglietto A, Secco GG, et al. Reduced rate of hospital admissions for ACS during COVID-19 outbreak in Northern Italy. N Engl J Med. 2020;383(1):88-89.

6. Bhagavathula AS, Shehab A. The story of mysterious pneumonia and the response to deadly novel coronavirus (2019-nCoV): so far. NEMJ. 2020;1(1):7-10.

7. Reinstadler SJ, Reindl M, Lechner I, Holzknecht M, Tiller C, Roithinger FX, Frick M, et al. Effect of the COVID-19 pandemic on treatment delays in patients with ST-segment elevation myocardial infarction. J Clin Med. 2020;9(7):2183.

8. Coughlan JJ, Chongprasertpon N, Arockiam S, Arnous S, Kiernan TJ. COVID-19 and STEMI: A snapshot analysis of presentation patterns during a pandemic. Int J Cardiol Heart Vasc. 2020;30:100546.

9. Kiss P, Carcel C, Hockham C, Peters SAE. The impact of the COVID-19 pandemic on the care and management of patients with acute cardiovascular disease: a systematic review. Eur Heart J Qual Care Clin Outcomes. 2021;7(1):18-27.

10. Wilson SJ, Connolly MJ, Elghamry Z, Cosgrove C, Firoozi S, Lim P, Sharma R, et al. Effect of the COVID-19 pandemic on ST-Segment-elevation myocardial infarction presentations and in-hospital outcomes. Circ Cardiovasc Interv. 2020;13(7):e009438.

11. Balghith MA. The Impact of COVID-19 Pandemic on the presentation and hospital management of STEMI patients in a tertiary care center in Saudi Arabia. Heart Views. 2020;21(3):166-170.

12. Wu J, Mamas M, Rashid M, Weston C, Hains J, Luescher $\mathrm{T}$, de Belder MA, et al. Patient response, treatments, and mortality for acute myocardial infarction during the COVID-19 pandemic. Eur Heart J Qual Care Clin Outcomes. 2021;7(3):238-246.

13. Rodriguez-Leor O, Cid-Alvarez B, Ojeda S, Martin-Moreiras J, Rumoroso JR, Lopez-Palop R, Serrador A, et al. Impact of the COVID-19 pandemic on interventional cardiology activity in Spain. REC IntervCardiol. 2020;2:82-89.

14. Metzler B, Siostrzonek P, Binder RK, Bauer A, Reinstadler SJ. Decline of acute coronary syndrome admis- 
sions in Austria since the outbreak of COVID-19: the pandemic response causes cardiac collateral damage. Eur Heart J. 2020;41(19):1852-1853.

15. Zitelny E, Newman N, Zhao D. STEMI during the COVID-19 pandemic - an evaluation of incidence. Cardiovasc Pathol. 2020;48:107232.

16. Daoulah A, Hersi AS, Al-Faifi SM, Alasmari A, Aljohar A, Balghith M, Alshehri M, et al. STEMI and COVID-19 pandemic in Saudi Arabia. Curr Probl Cardiol. 2021;46(3):100656.

17. Chew NW, Sia CH, Wee HL, Benedict LJ, Rastogi S, Kojodjojo P, Chor WPD, et al. Impact of the COVID-19 pandemic on door-to-balloon time for primary percutaneous coronary intervention- results from the Singapore Western STEMI Network. Circ J. 2021;85(2):139-149.

18. Butala NM, Patel NK, Chhatwal J, Vahdat V, Pomerantsev EV, Albaghdadi M, Sakhuja R, et al. Patient and provider risk in managing st-elevation myocardial infarction during the COVID-19 pandemic: a decision analysis. Circ Cardiovasc Interv. 2020;13(11):e010027.

19. Mafham MM, Spata E, Goldacre R, Gair D, Curnow P, Bray M, Hollings S, et al. COVID-19 pandemic and admission rates for and management of acute coronary syndromes in England. Lancet. 2020;396(10248):381-389. 